\title{
Spatial patterns of tau deposition are associated with amyloid, ApoE, sex, and cognitive decline in older adults
}

\author{
Joana B. Pereira ${ }^{1,5}$ (D) $\cdot$ Theresa M. Harrison ${ }^{2} \cdot$ Renaud La Joie $^{3} \cdot$ Suzanne L. Baker ${ }^{4}$. William J. Jagust ${ }^{2,4}$
}

Received: 7 September 2019 / Accepted: 23 December 2019 / Published online: 8 January 2020

(C) The Author(s) 2020

\begin{abstract}
Purpose The abnormal deposition of tau begins before the onset of clinical symptoms and seems to target specific brain networks. The aim of this study is to identify the spatial patterns of tau deposition in cognitively normal older adults and assess whether they are related to amyloid- $\beta(\mathrm{A} \beta), A P O E$, sex, and longitudinal cognitive decline.

Methods We included 114 older adults with cross-sectional flortaucipir (FTP) and Pittsburgh Compound-B PET in addition to longitudinal cognitive testing. A voxel-wise independent component analysis was applied to FTP images to identify the spatial patterns of tau deposition. We then assessed whether tau within these patterns differed by A $\beta$ status, APOE genotype, and sex. Linear mixed effects models were built to test whether tau in each component predicted cognitive decline. Finally, we ordered the spatial components based on the frequency of high tau deposition to model tau spread.

Results We found 10 biologically plausible tau patterns in the whole sample. There was greater tau in medial temporal, occipital, and orbitofrontal components in $\mathrm{A} \beta$-positive compared with $\mathrm{A} \beta$-negative individuals; in the parahippocampal component in $\varepsilon 3 \varepsilon 3$ compared with $\varepsilon 2 \varepsilon 3$ carriers; and in temporo-parietal and anterior frontal components in women compared with men. Higher tau in temporal and frontal components predicted longitudinal cognitive decline in memory and executive functions, respectively. Tau deposition was most frequently observed in medial temporal and ventral cortical areas, followed by lateral and primary areas.

Conclusions These findings suggest that the spatial patterns of tau in asymptomatic individuals are clinically meaningful and are associated with $\mathrm{A} \beta, A P O E \varepsilon 2 \varepsilon 3$, sex and cognitive decline. These patterns could be used to predict the regional spread of tau and perform in vivo tau staging in older adults.
\end{abstract}

Keywords Flortaucipir PET $\cdot$ Older adults $\cdot$ Amyloid $\cdot$ APOE $\cdot$ Sex $\cdot$ Cognitive decline

This article is part of the Topical Collection on Neurology

Electronic supplementary material The online version of this article (https://doi.org/10.1007/s00259-019-04669-x) contains supplementary material, which is available to authorized users.

Joana B. Pereira

joana.pereira@ki.se

1 Division of Clinical Geriatrics, Department of Neurobiology, Care Sciences and Society, Karolinska Institute, Stockholm, Sweden

2 Helen Wills Neuroscience Institute, University of California Berkeley, Berkeley, CA, USA

3 Memory and Aging Center, University of California, Oakland, CA, USA

4 Molecular Biophysics and Integrated Bioimaging, Lawrence Berkeley National Laboratory, Berkeley, CA, USA

5 Clinical Memory Research Unit, Department of Clinical Sciences, Lund University, Malmö, Sweden

\section{Background}

Alzheimer's disease (AD) is characterized by a long-lasting preclinical phase, in which the presence of progressively increasing pathology is not yet accompanied by clinical symptoms [1]. In this preclinical phase, biomarkers of tau pathology have important prognostic value and could be used to predict cognitive decline and identify individuals at higher risk of developing AD [2]. Thanks to the recent development of tau PET tracers, it is now possible to measure the regional distribution and topological patterns of tau, and assess how they are related to other biomarkers of early $\mathrm{AD}$ [3]. However, so far, most tau PET studies in older adults have focused on a single brain area or independent regions of interest, despite growing evidence that tau deposits in different areas are not independent from each other but are strongly correlated, forming spatial patterns or covariance networks [4-8]. 
The relationship between the spatial patterns of tau and biomarkers associated with risk of developing $A D$ remain unexplored in older adults. In particular, the effects of $A \beta$ positivity, $A P O E$, sex, and cognition on these patterns have not yet been assessed. In addition, no studies have examined tau PET differences between $A P O E \varepsilon 2 \varepsilon 3$ and $\varepsilon 3 \varepsilon 3$ carriers [9, $10]$. In this study, we sought to address these issues by identifying the spatial patterns of tau in older adults and assessing whether tau in these patterns is related to amyloid, $A P O E$, sex, or longitudinal cognitive decline. We also sorted the spatial patterns based on the frequency of tau positivity across subjects to determine a proxy for regional tau spread.

\section{Methods}

\section{Participants}

One hundred and fourteen cognitively normal individuals $\geq$ 60 years of age were included from the Berkeley Aging Cohort Study. All individuals underwent structural MRI, Pittsburgh Compound-B PET (PiB), and Flortaucipir (FTP) PET imaging in addition to neuropsychological testing. Inclusion criteria included a baseline Mini-Mental State Examination (MMSE) score $\geq 25$; normal performance on cognitive tests; no neurological, psychiatric, or major medical illness; no medications affecting cognition; and no imaging contraindications, and that all participants were community-dwelling.

\section{Neuropsychological test battery}

Eighty-six of the 114 subjects underwent longitudinal neuropsychological testing before and up to 1 year after the date of the FTP scan. These subjects had between 2 and 11 cognitive testing sessions (mean, $3.8 \pm 2.4$ ) over a period of $4.6 \pm$ 2.9 years, with an average delay of $1.2 \pm 0.4$ years between sessions. For each testing session, composite $Z$ scores for memory and executive function domains were created, as previously described [11]. These $Z$ scores were calculated using the means and standard deviations from the first cognitive session data of a larger sample of 150 healthy participants from the Berkeley Aging Cohort Study that also included the 86 older adults studied here. The memory composite score included the short- and long-delay free recall tests of the California Verbal Learning Test [12] and the Visual Reproduction test of the Wechsler Memory scale [13]. The executive function composite score included the Digit-Symbol test [14], Trail Making Test B minus A [15], and Stroop number of correct words in $1 \mathrm{~min}$ [16].

\section{APOE genotyping}

The determination of $A P O E$ alleles was performed using a TaqMan Allelic Discrimination Assay using a Real-Time
PCR system (Applied Biosystems, Foster City, CA), as previously described [17]. One subject had missing APOE information.

\section{Image acquisition}

All PET scans were acquired at the Lawrence Berkeley National Laboratory on a Siemens (Erlangen, Germany) Biograph 6 Truepoint PET/computed tomography (CT) scanner in 3D acquisition mode. FTP was synthesized using a TracerLab FXN-Pro (GE Medical Systems, Milwaukee, WI) synthesis module with a modified protocol based on an Avid Radiopharmaceuticals (Philadelphia, PA) protocol. Participants were injected with $10 \mathrm{mCi}$ of tracer and scanned in list mode from 75 to $105 \mathrm{~min}$ post-injection. Data was subsequently reconstructed as $4 \times 5$-min frames within 80 to 100 min post-injection. $\mathrm{PiB}$ was also synthesized according to a previously published protocol [18]. Immediately after the intravenous injection of approximately $15 \mathrm{mCi}$ of $\mathrm{PiB}$, 90 min of dynamic acquisition frames were obtained $(4 \times 15$, $8 \times 30,9 \times 60,2 \times 180,10 \times 300$, and $2 \times 600$ s). Both FTP and $\mathrm{PiB}$ images were reconstructed using an ordered subset expectation maximization algorithm with weighted attenuation and smoothed with a 4-mm Gaussian kernel with scatter correction (image resolution $=6.5 \times 6.5 \times 7.25 \mathrm{~mm}^{3}$ ).

T1-weighted structural MRI images were acquired on a 1.5-T Siemens Magnetom.

Avanto scanner using a magnetization-prepared rapid gradient echo sequences with the following parameters: repetition time $=2110 \mathrm{~ms}$, echo time $=3.58 \mathrm{~ms}$, flip angle $=15^{\circ}$, voxel size $=1 \mathrm{~mm}$ isotropic.

\section{Image preprocessing}

Both PiB and FTP PET frames were motion-corrected, timeaveraged, and coregistered to their T1-weighted images. For $\mathrm{PiB}$ images, distribution volume ratios (DVRs) were calculated using Logan graphical analysis on PiB frames corresponding to 35 to $90 \mathrm{~min}$ post-injection using all the frontal, temporal, and parietal regions provided by the Desikan-Killiany atlas of FreeSurfer as well as a cerebellar gray matter reference region $[19,20]$. Subjects were classified as $A \beta$-positive based on a mean global cortical DVR > 1.065 [21, 22].

For FTP, standardized uptake value ratio (SUVR) images were created using the mean inferior cerebellar gray matter uptake as the reference region [23-25]. These images were warped to MNI152 space using FSL (https://fsl.fmrib.ox.ac. $\mathrm{uk} / \mathrm{fs} / /$ fslwiki) and smoothed using an 8-mm Gaussian filter. Warping to MNI was performed using the transformation parameters derived from warping the T1-weighted images to the MNI152 template. The average delay between the PiB and FTP scans was $24.8 \pm 99.2$ days. 
In this study, we did not apply partial volume corrections (PVC) to the FTP data because methods suitable for voxelwise analyses fail to account for tracer binding in the multiple brain compartments (CSF, white matter, extracranial uptake) that can affect FTP images.

\section{Tau PET independent component analysis}

FTP PET images were submitted to an independent component analysis using the group ICA of functional MRI Toolbox (GIFT) [26]. This data-driven analysis decomposes the data into independent components or spatial patterns of correlated signals from distributed brain areas across subjects. To determine the optimal number of components, we used the minimum description length criterion [27]. In addition, in order to produce reliable components, we used the ICASSO algorithm implemented in GIFT, which ran ICA 10 times before selecting stable components that corresponded to tight clusters. A brain mask that excluded the basal ganglia, brainstem, cerebellum, subcortical white matter, and cerebrospinal fluid (including the choroid plexus) was included in the analyses to minimize contamination from regions considered to represent off-target binding in FTP data [28-30]. After identifying the spatial patterns, we thresholded them with a $Z$ score $>2.0$, which corresponds to a two-tailed significance value of $p<0.05$.

\section{Statistical analyses}

Differences in baseline characteristics between groups were analyzed using chi-squared tests for binary variables and analyses of variance for continuous variables. To assess whether tau burden within each spatial component differed by $\mathrm{A} \beta$ status, $A P O E$ genotype and sex, we compared mean SUVRs within these components between $\mathrm{A} \beta-$ and $\mathrm{A} \beta+$ groups, subjects with different $A P O E$ genotypes, and male and female participants. These analyses were carried out using permutation tests with 1000 replicates, while controlling for age, sex, education, and PiB DVR, when appropriate. To identify and exclude potential outliers in tau SUVRs, we applied the Tukey method $(1.5 \times$ interquartile range). Adjustment for multiple comparisons was performed using false discovery rate (FDR) corrections [31] at $q<0.05$. For all significant group differences, we calculated effect sizes using Cohen's $d$ [32].

To evaluate whether the spatial patterns of tau were associated with change in cognition over time, we fitted linear mixed effect models, implemented in R using "Ime4." In these models, we included memory or executive scores as dependent variables, and tau component SUVRs, time since first cognitive evaluation, age, sex, education, and PiB DVR as fixed effects. These models included all main effects, the interaction between tau SUVRs and time, and random effects for intercepts and slopes: cognitive scores $\sim$ time $\times$ tau network SUVR + age + sex + education + PIB DVR + (time|subject). We built separate models for each tau component.

Finally, to determine the order of tau spreading across the different components, we selected $A \beta$ - individuals whose FTP SUVR values in the entorhinal cortex were $<1.2$ to obtain regional mean and standard deviation values of SUVR, following a previously described procedure [33]. Using these means and standard deviations, we computed $Z$ scores for the mean SUVR in each tau component across all participants. For each participant, components with a $Z$ score $>2.5$ were considered to be positive for tau deposition. Using this approach, the frequency of positive tau deposition was assessed for each component. Components were then ordered from highest to lowest frequency since we assumed that components with a higher frequency of positive tau deposition comprised earlier accumulating regions. This approach allowed us to estimate spatial tau spread using cross-sectional data.

\section{Results}

\section{Participants}

Characteristics of the sample are summarized in Table 1. The majority of participants had one of the following APOE genotypes: $\varepsilon 2 \varepsilon 3(n=11), \varepsilon 3 \varepsilon 3(n=71)$, or $\varepsilon 3 \varepsilon 4(n=28)$. In addition, 3 participants were $\varepsilon 2 \varepsilon 4$ carriers, which were excluded from the analyses due to the small number of subjects in this group.

In this study, $\mathrm{A} \beta$-positive individuals were less educated $(d=$ $0.4, p=0.031)$ and had a higher prevalence of the APOE $\varepsilon 3 \varepsilon 4$ genotype $(d=1.1, p<0.001)$ than their $\mathrm{A} \beta$-negative peers. As expected, $\varepsilon 3 \varepsilon 4$ carriers showed higher PiB DVRs compared with both $\varepsilon 2 \varepsilon 3(d=1.4, p=0.004)$ and $\varepsilon 3 \varepsilon 3(d=0.9$, $p<0.001)$ carriers, and $\varepsilon 2 \varepsilon 3$ were less educated than $\varepsilon 3 \varepsilon 3$ carriers $(d=0.7, p=0.003)$. In our sample, men had lower executive function scores compared with women $(d=0.5, p=0.025)$.

\section{Spatial patterns of tau deposition in older adults}

The optimal number of components identified in FTP data was 15 , which explained $85.2 \%$ of the total variance. Five of these components were excluded from the analyses since they had white matter voxels or consisted of thin clusters surrounding the meninges, which is an off-target binding region for ${ }^{18}$ F-FTP data. The excluded components can be found in Supplementary Fig. 1. After excluding the noisy components, the total variance explained by the data was $48.5 \%$. This was due to the fact that one of the excluded components explained a high proportion of the variance or a wide spread in the FTP values in our dataset $(22.9 \%)$. This component was characterized by high tau signal in a thin elongated cluster surrounding the meninges (component 4 in Supplementary Fig. 1). The meninges have been previously described as an off-target 
Table 1 Characteristics of the sample

\begin{tabular}{|c|c|c|c|c|c|c|c|c|}
\hline & $\begin{array}{l}\text { All subjects } \\
(n=114)\end{array}$ & $\mathrm{A} \beta-(n=67)$ & $\mathrm{A} \beta+(n=47)$ & $\begin{array}{l}\text { ApoE } \varepsilon 2 / \varepsilon 3 \\
(n=11)\end{array}$ & $\begin{array}{l}\text { ApoE } \varepsilon 3 / \varepsilon 3 \\
(n=71)\end{array}$ & $\begin{array}{l}\text { ApoE } \varepsilon 3 / \varepsilon 4 \\
(n=28)\end{array}$ & $\operatorname{Men}(n=45)$ & $\begin{array}{l}\text { Women } \\
(n=69)\end{array}$ \\
\hline Age (years) & $76.4(6.3)$ & $76.3(7.4)$ & $76.6(4.3)$ & $75.2(7.6)$ & $76.4(6.8)$ & $76.6(4.6)$ & $77.1(6.7)$ & $75.9(6.0)$ \\
\hline $\operatorname{Sex}(m / f)$ & $45 / 69$ & $27 / 40$ & $18 / 29$ & $7 / 4$ & $25 / 46$ & $12 / 16$ & - & - \\
\hline $\begin{array}{l}\text { Education } \\
\quad \text { (years) }^{\mathrm{a}, \mathrm{b}}\end{array}$ & $16.8(1.8)$ & $17.2(1.8)$ & $16.4(1.8)$ & $16.3(0.6)$ & $17.2(1.7)$ & $16.4(2.3)$ & $17.1(1.6)$ & $16.7(2.0)$ \\
\hline PiB DVR ${ }^{a, b, c, d}$ & $1.2(0.2)$ & $1.02(0.0)$ & $1.34(0.3)$ & $1.02(0.04)$ & $1.09(0.16)$ & $1.30(0.3)$ & $1.12(0.2)$ & $1.17(0.2)$ \\
\hline $\begin{array}{l}\text { APOE } \\
\quad(\mathrm{e} 2 / \mathrm{e} 3 / \mathrm{e} 4)^{\mathrm{a}}\end{array}$ & $11 / 71 / 28$ & $10 / 52 / 5$ & $1 / 19 / 23$ & - & - & - & $7 / 25 / 12$ & $4 / 46 / 16$ \\
\hline MMSE & $28.8(1.2)$ & $28.9(1.0)$ & $28.5(1.4)$ & $29.2(0.8)$ & $28.8(1.2)$ & $28.5(1.3)$ & $28.8(1.2)$ & $28.7(1.2)$ \\
\hline Memory & $0.02(0.9)$ & $0.14(0.9)$ & $-0.15(0.9)$ & $0.54(0.9)$ & $-0.04(0.9)$ & $0.10(0.8)$ & $-0.08(0.8)$ & $0.09(1.0)$ \\
\hline $\begin{array}{l}\text { Executive } \\
\text { function }^{\mathrm{e}}\end{array}$ & $0.06(0.7)$ & $0.11(0.7)$ & $-0.01(0.7)$ & $0.17(0.9)$ & $0.01(0.7)$ & $0.22(0.7)$ & $-0.12(0.6)$ & $0.18(0.8)$ \\
\hline
\end{tabular}

Values in the table correspond to means followed by (standard deviation), except for sex and APOE, which correspond to number of subjects in each category

${ }^{\mathrm{a}}$ Significant differences between $\mathrm{A} \beta-$ and $\mathrm{A} \beta+$ groups $(p<0.05)$

${ }^{\mathrm{b}}$ Significant differences between $\mathrm{ApoE} \varepsilon 2 / \varepsilon 3$ and $\mathrm{ApoE} \varepsilon 3 / \varepsilon 3$ groups $(p<0.05)$

${ }^{\mathrm{c}}$ Significant differences between ApoE $\varepsilon 2 / \varepsilon 3$ and ApoE $\varepsilon 3 / \varepsilon 4$ groups $(p<0.05)$

${ }^{\mathrm{d}}$ Significant differences between ApoE $\varepsilon 3 / \varepsilon 3$ and ApoE $\varepsilon 3 / \varepsilon 4$ groups $(p<0.05)$

${ }^{\text {e }}$ Significant differences between men and women $(p<0.05)$

binding area for FTP as well as other tau PET tracers. Importantly, their signal varies across subjects and can potentially bleed into cortical areas $[34,35]$. Our independent component analyses were able to identify this source of variable off-target binding, which allowed us to effectively remove it from our main analyses. The variance explained by each of the 15 components can be found in Supplementary Table 1.

After excluding the noisy components, the remaining components $(n=10)$ were included in further analyses. These components involved bilateral gray matter regions, except for 2, which were unilateral (Fig. 1). The bilateral components included medial temporal, parahippocampal, superior and anterior frontal, lateral temporo-occipital, orbitofrontal, parietal, and sensorimotor areas. All of these components were symmetric, with the exception of the lateral temporo-parietal component, which showed greater involvement on the left hemisphere. The unilateral components included left and right inferior occipital areas. The distribution of tau SUVRs in these components can be found in Supplementary Fig. 2.

\section{Tau burden in spatial patterns differs by $A \beta$ status, $A P O E$ genotype and sex}

Our group analyses showed that $\mathrm{A} \beta$-positive individuals had higher tau SUVRs within the medial temporal $(d=0.8$, $p<0.001)$, parahippocampal $(d=0.5, p=0.008)$, left and right inferior occipital ( $d=0.5, p=0.005 ; d=0.5, p=0.003)$, lateral temporo-parietal $(d=0.5, p=0.001)$ and orbitofrontal $(d=0.5, p=0.008)$ components compared with $\mathrm{A} \beta$-negative subjects (Fig. 2a), after FDR corrections.
In addition, we found higher tau SUVRs in the parahippocampal component $(d=0.7, p=0.008)$ in $\varepsilon 3 \varepsilon 3$ carriers compared with those in $\varepsilon 2 \varepsilon 3$ carriers after adjusting for PiB DVR and for multiple comparisons (Fig. 2b). When PiB was excluded as a covariate from these analyses, we observed tau increases in additional networks in $\varepsilon 3 \varepsilon 3$ and $\varepsilon 3 \varepsilon 4$ compared with $\varepsilon 2 \varepsilon 3$ carriers (Supplementary Table 2).

Finally, our analyses comparing male and female participants showed that women had higher tau in the anterior frontal $(d=$ $0.5, p=0.007)$ and lateral temporo-parietal $(d=0.6, p<0.001)$ components compared with men (Fig. 2c) after adjusting for PiB and FDR. These differences were still significant after excluding $\mathrm{PiB}$ as a covariate (Supplementary Table 2).

\section{Spatial tau patterns are associated with longitudinal cognitive decline}

Our mixed effects models did not show any significant interactions between time and the different tau patterns in cognitive decline after adjusting for multiple comparisons with FDR $(p<0.05)$. However, at an uncorrected level, we identified an interaction between time and the parahippocampal (estimate, 0.15; SD, 0.05; $t$ score, $-2.891, p=0.007$ ), left inferior occipital (estimate, $-0.20 ; \mathrm{SD}, 0.08 ; t$ score, $-2.613, p=0.010$ ), and lateral temporo-parietal (estimate, -0.17 ; SD, $0.08 ; t$ score, 2.201, $p=0.032$ ) components (Supplementary Table 3), indicating that participants with higher tau within these patterns had worse longitudinal decline in memory. In addition, we also found an interaction between the superior frontal tau pattern and time (estimate, -0.13 ; SD, 0.06; $t$ score, $-2.305, p=0.024$ ) 

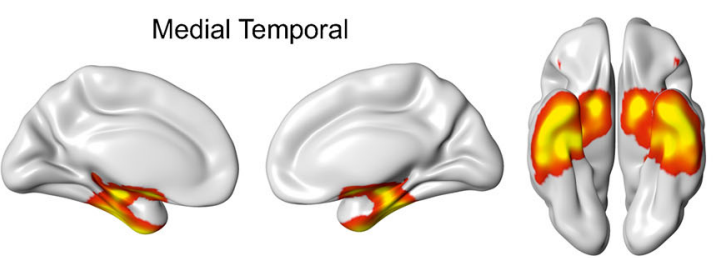

Left Inferior Occipital
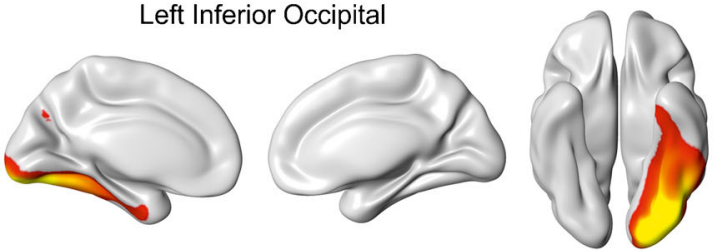

Superior Frontal
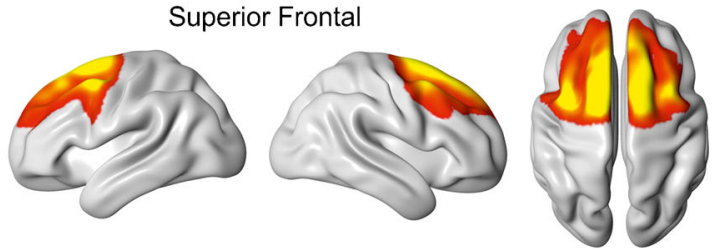

Lateral Temporo-Parietal
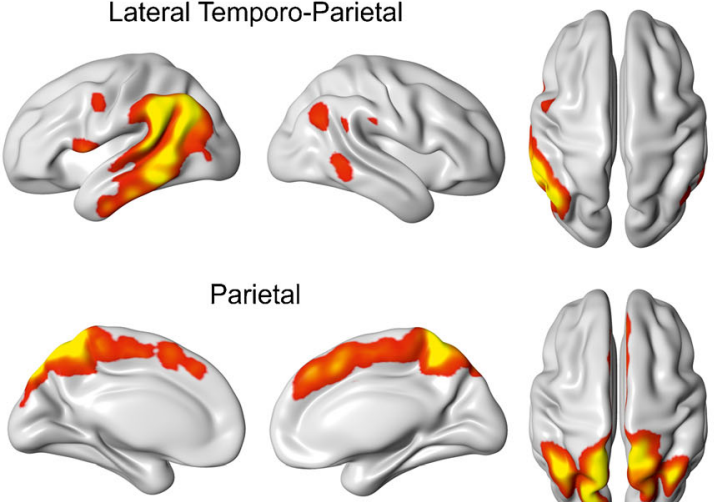

Parietal
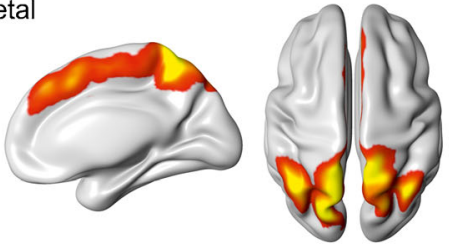

2.0
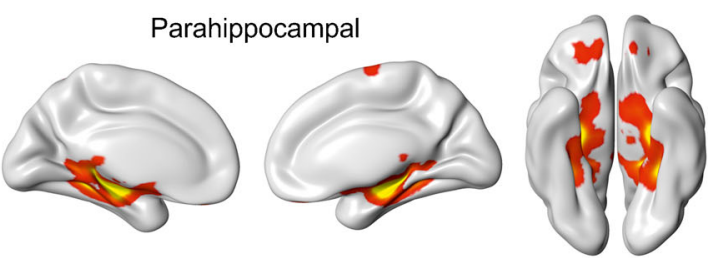

Right Inferior Occipital
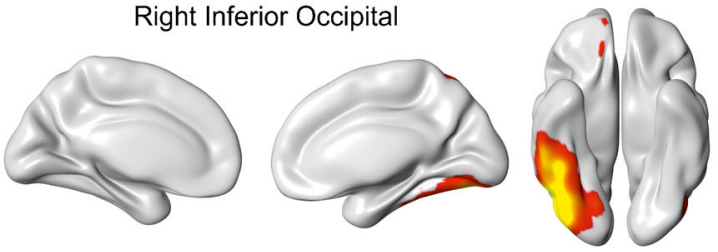

Anterior Frontal
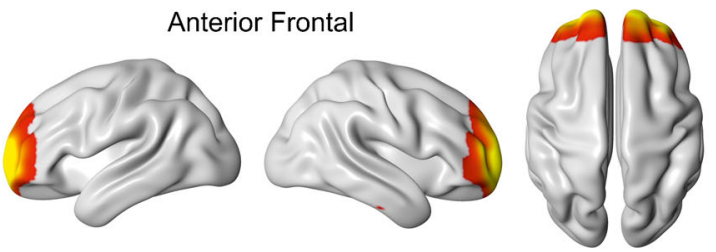

Orbitofrontal
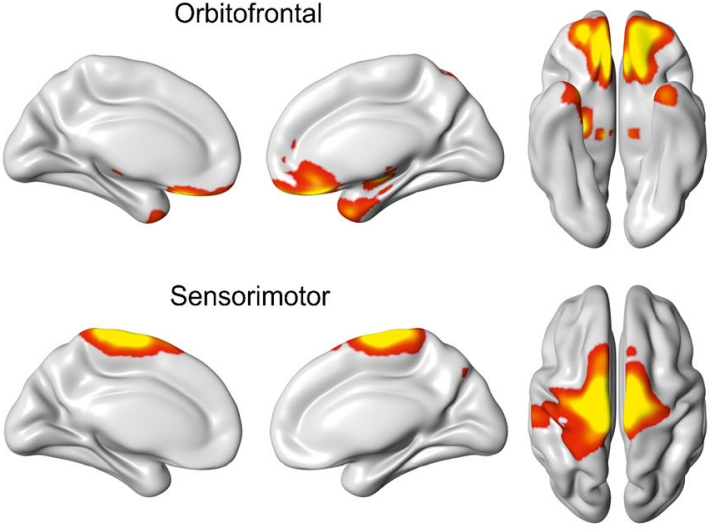

Fig. 1 Spatial patterns of tau deposition in aging. We identified 10 independent components for tau in the analyses of FTP data. These components were thresholded with a $Z$ score $>2.0$

(Supplementary Table 3), indicating that participants with higher tau within this pattern had worse executive decline.

We also ran additional analyses using linear mixed effects models that included interactions between time and all covariates (age, sex, education, PIB DVR). These models showed lower AIC and BIC values than the models with a single interaction term between time and tau (Supplementary Table 4), indicating they were a worse fit to our data.

\section{In vivo staging of tau spatial patterns}

The progression of tau pathology across the spatial patterns is displayed in Fig. 3, and a table of overlaps of high tau across the patterns in different subjects is provided in Supplementary Table 5. In the whole cohort, we found that tau deposition was most frequently observed in the parahippocampal $(25.4 \%, n=$ 29) pattern (I). This was followed by regional involvement of ventral areas that included the left inferior occipital $(15.8 \%, n=$ 18) (II), right inferior occipital $(12.3 \%, n=14)$, orbitofrontal $(12.3 \%, n=14)$ (III), and medial temporal (11.4\%, $n=13)$ (IV) components. Once most of the ventral areas were affected by tau pathology, the more frequent set of regions with high tau deposition included lateral and medial cortical areas such as the lateral temporo-parietal $(6.1 \%, n=7)(\mathrm{V})$, parietal $(5.3 \%, n=6)(\mathrm{VI})$, and superior frontal $(3.5 \%, n=4)$ (VII) components. Finally, the least affected patterns with tau were the sensorimotor $(2.6 \%, n=$ $3)$ (VIII) and anterior frontal $(1.8 \%, n=2)$ (IX) components.

\section{Discussion}

With upcoming therapeutic approaches based on tau pathology, new tau biomarkers are urgently needed for early AD [36]. In this study, we show that the spatial patterns of tau 
Fig. 2 Tau burden within spatial patterns differs by $\mathrm{A} \beta, A P O E$, and sex in older adults. We found significantly higher tau burden in some of the spatial patterns in $\mathrm{A} \beta$-positive compared with $\mathrm{A} \beta$ negative peers, $A P O E \varepsilon 3 \varepsilon 3$ compared with $\varepsilon 2 \varepsilon 3$ carriers, and in women compared with men. Asterisk indicates significant differences between groups, after regressing out the effects of age, sex, education for $A \beta$ groups; age, sex, education, and PiB DVR for $A P O E$ groups; and age, education, and PiB DVR for sex groups. All results were corrected for multiple comparisons using FDR $(q<0.05)$ a $A \beta$

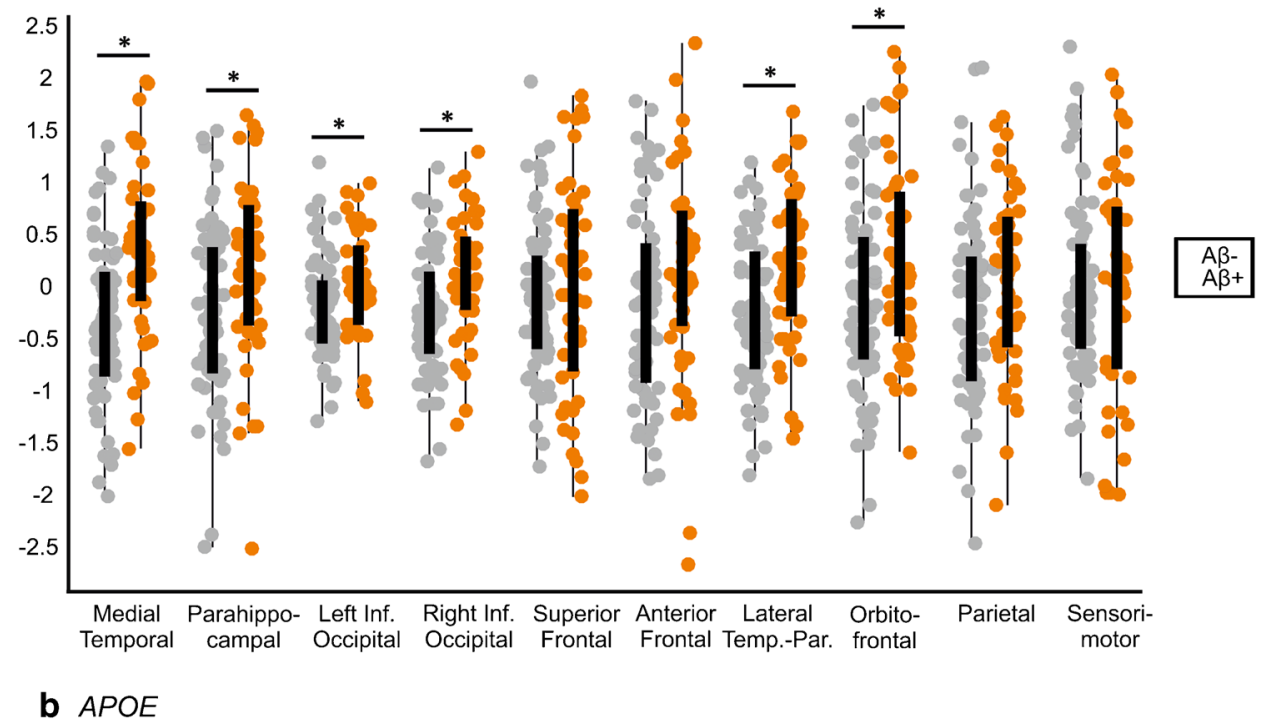

C Sex

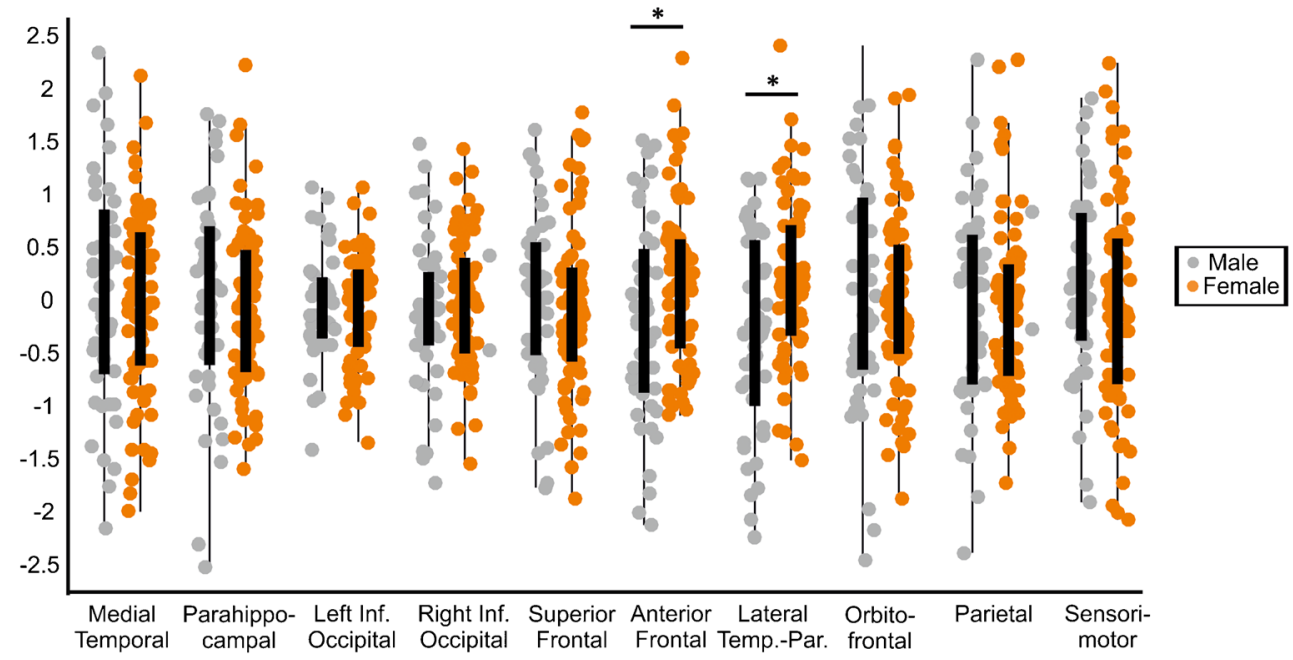

deposition could potentially be used as early biomarkers of $\mathrm{AD}$ and detect changes in clinically normal older individuals who are $\mathrm{A} \beta$-positive, $A P O E \varepsilon 3 \varepsilon 3$ carriers, or women. In addition, the association we found between some of these 

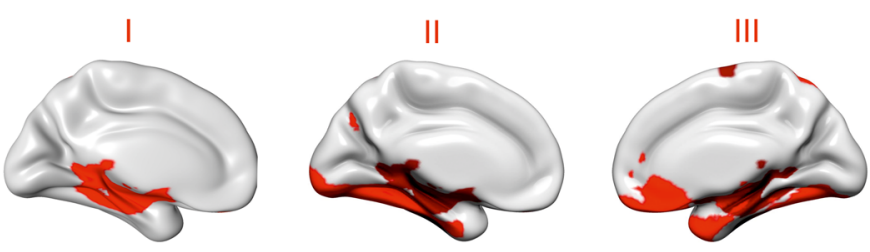

IV
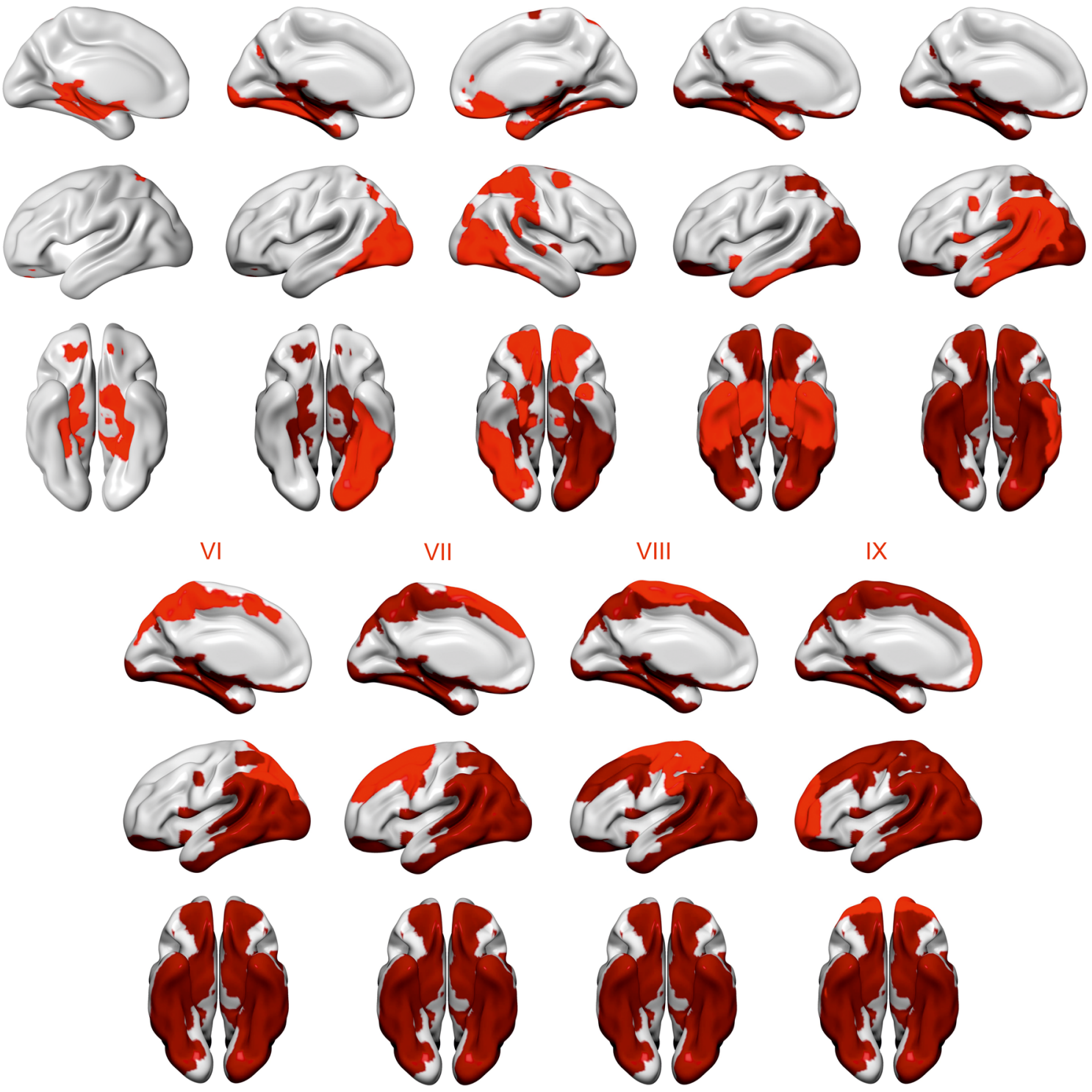

Fig. 3 Progression of tau pathology across different spatial patterns. We used the means and standard deviations of $A \beta-$ individuals with entorhinal tau $<1.2$ to identify individuals with high tau in the different spatial patterns. The patterns that were determined to show increased binding in a greater number of participants were considered to be regions of early tau deposition. Using this approach, we found a

patterns and cognitive decline suggest they could also be used to understand the variability in longitudinal cognitive trajectories among older adults.

There is increasing evidence showing that, rather than appearing in unrelated brain regions, tau pathology occurs within the boundaries of distributed networks [37, 38]. However, these networks have been poorly characterized in cognitively normal individuals, with only one study identifying four clusters of areas with tau deposition [8]. Our datadriven approach detected 10 independent spatial patterns of tau across temporal (medial temporal, parahippocampal, lateral temporo-parietal), occipital (left and right inferior occipital),

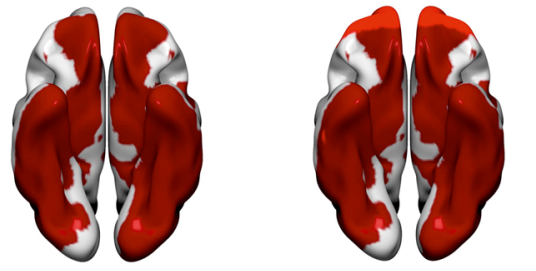

spreading order of tau across the spatial patterns from limbic and ventral areas, to lateral and medial posterior regions, and frontal and sensorimotor cortices. Patterns colored in light red correspond to a newly added stage, whereas patterns colored in dark red correspond to

frontal (orbitofrontal, middle frontal, anterior frontal), parietal, and sensorimotor regions. This finding might seem surprising, as several studies have shown that tau is either absent or restricted to temporal regions in cognitively normal individuals [39-41]. Our study shows that an unsupervised network approach may reveal additional patterns in tau PET data, which can be used to understand why some individuals, despite being clinically normal, are more prone to accumulate tau in specific brain areas. For instance, it has been previously shown that $A \beta$-positive individuals show higher tau deposition than their $A \beta$-negative counterparts [40-42] due to some interaction between amyloid and tau that is not fully previous stage 
understood [42]. Here, we show that this relationship can be observed not only in temporal areas but also in inferior occipital, lateral temporo-parietal, and orbitofrontal regions, confirming that $\mathrm{A} \beta$ may accelerate the spread of tau deposition outside the temporal cortex into adjacent areas [42]. In addition to $A \beta$, the role of sex in tau deposition has been recently assessed in clinically normal adults, with women showing greater temporal tau than men in the presence of high $A \beta$ burden [43]. In our study, we found that women had greater tau in temporo-parietal and anterior frontal components compared with men, indicating that the effects of sex on tau deposition might be more widespread than previously thought. The higher AD prevalence previously reported in women [44] together with evidence of heightened female inflammatory responses [45] and relationship between tauopathy and menopause [46] could potentially explain why women are more vulnerable to tau deposition than men. Future studies are needed to determine the exact biological underpinnings of sex differences in tau deposition. Finally, to our knowledge, our study is the first showing the protective value of the $A P O E \varepsilon 2$ allele against in vivo tau deposition in clinically normal adults. We found that the $\varepsilon 2 \varepsilon 3$ genotype was associated with lower tau in parahippocampal areas compared with the more frequent $\varepsilon 3 \varepsilon 3$ genotype, before and after controlling for $A \beta$. In contrast, we found that the increases of tau load in $\varepsilon 3 \varepsilon 4$ carriers were mostly mediated by $A \beta$. Our results agree with previous findings of reduced neurofibrillary tangle pathology in $\varepsilon 2$ carriers [47], and the well-known associations between amyloid APOE $\varepsilon 4$ and amyloid [48], which are not independent from each other.

One of the earliest cognitive changes in aging, and the one that has probably received most attention due to its association with $\mathrm{AD}$, is the decline of memory. Here we found that higher tau load in the parahippocampal, left inferior occipital, and lateral temporo-parietal patterns predicted retrospective longitudinal decline in memory in older adults, although these associations did not remain significant after adjustment for multiple comparisons. These results potentially strengthen the hypothesis that tau pathology plays a key role in memory decline in aging [49], which is also strongly related to dysfunction of temporal and some parietal regions. In addition, we found that higher tau in the superior frontal pattern predicted retrospective decline in executive functions, a finding that has not yet been reported by previous studies. There is mounting evidence showing that the decline of executive functions is associated with dysfunction in frontal brain areas in aging [50]. Our findings provide evidence that this relationship might be at least partially mediated by tau and explain some of the variability observed in executive abilities in older adults [51]. However, this association did not survive FDR corrections and thus should be interpreted with caution.

In this study, to determine the "spread" of tau across the different spatial components, we calculated the frequency of high tau in each component in all participants. This approach showed that tau deposition was most frequent in the parahippocampus, followed by ventral areas such as the inferior temporal, inferior occipital, and orbitofrontal cortices. Then, from these ventral areas, tau spread into lateral temporoparietal and medial parietal regions, and finally reached frontal areas and the sensorimotor cortex. This regional progression of tau largely mirrors the spread of neurofibrillary tangles previously reported by Braak et al. [52]. Interestingly, by using spatial patterns of tau deposition, we were able to reproduce the spread of tau from transentorhinal areas to other ventral areas of the brain [52], suggesting that the spatial information of our components might be useful for in vivo staging of tau pathology. Our findings also largely agree with those reported by a previous study specifically assessing the regional spread of tau across regions of interest [33].

Our study has some limitations. Although comparable with previous tau PET studies, our sample size was modest and might have limited our ability to detect additional spatial patterns or their value in characterizing $\mathrm{A} \beta, A P O E$, and sex effects. In particular, the number of $\varepsilon 2 \varepsilon 3$ carriers was very small $(n=11)$, in agreement with the low prevalence of this genotype in the population [53]. Thus, our findings should be considered preliminary and replicated in future studies. In addition, not all individuals had the same number of longitudinal cognitive visits, something we dealt with using linear mixed effects models. Moreover, although we did our best to reduce partial volume effects by excluding regions known to have off-target binding in FTP data such as the basal ganglia, the choroid plexus, and the meninges [28-30], it is still possible that off-target binding could have added some noise to our analyses. Our data-driven approach based on independent component analyses has been previously described as a useful technique to separate signal from noise [54]. In fact, in our study, it was able to detect noisy components in thin areas surrounding the meninges and in the white matter, which were excluded from the analyses. In particular, the component surrounding the meninges explained a high percentage of the variance in our dataset (22.9\%), in line with previous evidence showing that several tau PET tracers bind strongly to the meninges [34, 35, 55], which tends to spill into adjacent cortical areas. The reason for this strong off-target binding in the meninges and adjacent areas is unknown but a previous study showed that it seems to be more frequent in individuals who do not exhibit specific binding in regions typically associated with neurofibrillary tangles [55] such as cognitively normal subjects without amyloid pathology, who constituted more than $50 \%$ of our sample. Thus, we hope that, by applying an independent component analysis to FTP data, we removed as much noise as possible from our analyses. Finally, the analyses involving cognition did not survive control for multiple comparisons probably due to the fact that the subjects of our sample were cognitive normal and did not show strong 
memory or executive decline over time. Future studies are needed that allow assessing the relationship between the tau patterns and longitudinal cognitive trajectories for several decades, during which greater cognitive changes are more likely to occur.

\section{Conclusions}

Our findings indicate that distinct spatial tau patterns are present in cognitively normal adults and $A \beta$-negativity, $\varepsilon 2 \varepsilon 3$ carriership, and male sex are protective factors against tau deposition within these patterns. In light of the increasing recognition of $\mathrm{AD}$ as a network disorder that affects distributed brain systems [55], we hope that our findings might encourage future studies to analyze tau PET data using multivariate approaches that can characterize tau pathology across brain networks.

Acknowledgments The authors thank all study participants for their contribution to this research.

Availability of data and material Data used in this study will be shared by request from qualified investigators subject to negotiation of a data use agreement.

Authors' contributions J.B.P. and T.H. contributed to the design of the study, analysis and interpretation of data, and the statistical analysis. R.L. and S.B. provided technical support. W.J. was responsible for obtaining funding. All authors contributed to revising the manuscript.

Funding information Open access funding provided by Karolinska Institute. This research was supported by the NIH National Institute on Aging grants F32-AG057107 (T.M.H) and R01-AG034570 (W.J.J). Support was also provided by the Tau Consortium (W.J.J), the Alzheimer's Association (AARF-16-443,577, R.L.J.), the Swedish Research Council (J.B.P.), and Alzheimerfonden (J.B.P.). Avid Radiopharmaceuticals enabled the use of the $\left[{ }^{18} \mathrm{~F}\right]$ FTP tracer, but did not provide direct funding and were not involved in data analysis or interpretation.

\section{Compliance with ethical standards}

Conflict of interest J.B.P., T.M.H., and R.L. report no disclosures relevant to the manuscript. S.B. serves as a consultant to Genentech. W.J. has served as a consultant to BioClinica, Genentech, and Novartis Pharmaceuticals.

Ethics approval and consent to participate The institutional review board at Lawrence Berkeley National Laboratory and the University of California, Berkeley, approved the project, and written, informed consent was obtained from all participants.

Open Access This article is licensed under a Creative Commons Attribution 4.0 International License, which permits use, sharing, adaptation, distribution and reproduction in any medium or format, as long as you give appropriate credit to the original author(s) and the source, provide a link to the Creative Commons licence, and indicate if changes were made. The images or other third party material in this article are included in the article's Creative Commons licence, unless indicated otherwise in a credit line to the material. If material is not included in the article's Creative Commons licence and your intended use is not permitted by statutory regulation or exceeds the permitted use, you will need to obtain permission directly from the copyright holder. To view a copy of this licence, visit http://creativecommons.org/licenses/by/4.0/.

\section{References}

1. Jack CR Jr, Wiste HJ, Weigand SD, Therneau TM, Knopman DS, Lowe V, et al. Age-specific and sex-specific prevalence of cerebral beta-amyloidosis, tauopathy, and neurodegeneration in cognitively unimpaired individuals aged 50-95 years: a cross-sectional study. Lancet Neurol. 2017;16:435-44.

2. Sperling RA, Aisen PS, Beckett LA, Bennett DA, Craft S, Fagan $\mathrm{AM}$, et al. Toward defining the preclinical stages of Alzheimer's disease: recommendations from the National Institute on AgingAlzheimer's association workgroups on diagnostic guidelines for Alzheimer's disease. Alzheimers Dement. 2011;7:280-92.

3. Villemagne VL, Fodero-Tavoletti MT, Masters CL, Rowe CC. Tau imaging: early progress and future directions. Lancet Neurol. 2015;14(1):114-24.

4. Brier MR, Gordon B, Friedrichsen K, McCarthy J, Stern A, Christensen $\mathrm{J}$ et al. Tau and A $\beta$ imaging, CSF measures, and cognition in Alzheimer's disease. Science Trans Med. 2016;8:338ra66-

5. Hoenig MC, Bischof GN, Seemiller J, Hammes J, Kukolja J, Onur ÖA, et al. Networks of tau distribution in Alzheimer's disease. Brain. 2018;141(2):568-81.

6. Jones DT, Graff-Radford J, Lowe VJ, Wiste HJ, Gunter JL, Senjem ML, et al. Tau, amyloid, and cascading network failure across the Alzheimer's disease spectrum. Cortex. 2017;97:143-59.

7. Sepulcre J, Schultz AP, Sabuncu M, Gomez-Isla T, Chhatwal J, Becker A, et al. In vivo tau, amyloid, and gray matter profiles in the aging brain. J Neurosci. 2016;36:7364-74.

8. Sepulcre J, Grothe MJ, Sabuncu M, Chhatwal J, Schultz AP, Hanseeuw B, et al. Hierarchical organization of tau and amyloid deposits in the cerebral cortex. JAMA Neurol. 2017;74:813-20.

9. Grothe MJ, Villeneuve S, Dyrba M, Bartrés-Faz D, Wirth M, Alzheimer's Disease Neuroimaging Initiative. Multimodal characterization of older APOE2 carriers reveals selective reduction of amyloid load. Neurology. 2017;88:569-576.

10. Suri S, Heise V, Trachtenberg AJ, Mackay CE. The forgotten APOE allele: a review of the evidence and suggested mechanisms for the protective effect of APOEe2. Neurosci Biobehav Rev. 2013;37:2878-86.

11. Harrison TM, Maass A, Baker SL, Harrison TM, Maass A, Baker SL, et al. Brain morphology, cognition, and $\beta$-amyloid in older adults with superior memory performance. Neurobiol Aging. 2018;67:162-70.

12. Delis DC, Freeland J, Kramer JH, Kaplan E. Integrating clinical assessment with cognitive neuroscience: construct validation of the California Verbal Learning Test. J Consult Clin Psychol. 1988;56:123.

13. Wechsler D. Wechsler memory scale. 1945.

14. Smith A. Symbol digit modalities test: manual. Western Psychological Services: Los Angeles, CA; 1982.

15. Tombaugh TN. Trail Making Test A and B: normative data stratified by age and education. Arch Clin Neuropsychol. 2004;19:20314.

16. Jensen AR. Scoring the Stroop test. Acta Psychol. 1965;24:398408.

17. Coppola G, Chinnathambi S, Lee JJ, Dombroski BA, Baker MC, Soto-Ortolaza AI, et al. Evidence for a role of the rare p. A152T 
variant in MAPT in increasing the risk for FTD-spectrum and Alzheimer's diseases. Hum Mol Gen. 2012;21(15):3500-12.

18. Mathis CA, Wang Y, Holt DP, Huang GF, Debnath ML, et al. Synthesis and evaluation of ${ }^{11} \mathrm{C}$ labeled 6-substituted 2arylbenzothiazoles as amyloid imaging agents. J Med Chem. 2003;46:2740-54.

19. Logan J, Fowler JS, Volkow ND, Wang GJ, Ding YS, Alexoff DL. Distribution volume ratios without blood sampling from graphical analysis of PET data. J Cereb Blood Flow Metab. 1996;16:834-40.

20. Price JC, Klunk WE, Lopresti BJ, Lu X, Hoge JA, Ziolko SK, et al. Kinetic modeling of amyloid binding in humans using PET imaging and Pittsburgh compound-B. J Cereb Blood Flow Metab. 2005;25: 1528-47.

21. Mormino EC, Brandel MG, Madison CM, Rabinovici GD, Marks S, Baker SL, et al. Not quite PIB-positive, not quite PIB-negative: slight PIB elevations in elderly normal control subjects are biologically relevant. Neuroimage. 2012;59:1152-60.

22. Villeneuve S, Rabinovici GD, Cohn-Sheehy BI, et al. Existing Pittsburgh compound-B positron emission tomography thresholds are too high: statistical and pathological evaluation. Brain. 2015;138:2020-33.

23. Shcherbinin S, Schwarz AJ, Joshi A, Madison C, Ayakta N, Ghosh PM, et al. Kinetics of the tau PET tracer 18FAV-1451 (T807) in subjects with normal cognitive function, mild cognitive impairment, and Alzheimer disease. J Nucl Med. 2016;57:1535-42.

24. Baker SL, Lockhart SN, Price JC, He M, Huesman RH, Schonhaut $\mathrm{D}$, et al. Reference tissue-based kinetic evaluation of ${ }^{18} \mathrm{~F}-\mathrm{AV}-1451$ in aging and dementia. J Nucl Med. 2017;52:332-8.

25. Wooten DW, Guehl NJ, Verwer EE, Shoup TM, Yokell DL, Zubcevik N, et al. Pharmacokinetic evaluation of the tau PET radiotracer $\left[{ }^{18} \mathrm{~F}\right] \mathrm{T} 807\left(\left[{ }^{18} \mathrm{~F}\right] \mathrm{AV}-1451\right)$ in human subjects. J Nucl Med. 2017;58:484-91.

26. Correa N, Adali T, Li YO, Calhoun VD. Comparison of blind source separation algorithms for FMRI using a new Matlab toolbox: GIFT. IEEE International Conference on Acoustics, Speech, and Signal Processing, 2005;5:401-.

27. Rissanen J. Modeling by shortest data description. Automatica. 1978;14:465-71.

28. Lockhart SN, Ayakta N, Winer JR, La Joie R, Rabinovici GD, Jagust WJ. Elevated 18F-AV-1451 PET tracer uptake detected in incidental imaging findings. Neurology. 2017;88:1095-7.

29. Choi JY, Cho H, Ahn SJ, Lee JH, Ryu YH, Lee MS, et al. Off-target $18 \mathrm{~F}-\mathrm{AV}-1451$ binding in the basal ganglia correlates with agerelated iron accumulation. J Nucl Med. 2018;59:117-20.

30. Baker SL, Harrison TM, Maßß A, La Joie R, Jagust W. Effect of offtarget binding on $18 \mathrm{~F}-$ Flortaucipir variability in healthy controls across the lifespan. J Nucl Med. 2019; jnumed-118.

31. Benjamini Y, Hochberg Y. Controlling the false discovery rate: a practical and powerful approach to multiple testing. J R Stat Soc Series B. 1995;1:289-300.

32. Cohen J. A power primer. Psychol Bull. 1992;112:155.

33. Cho H, Choi JY, Hwang MS, Kim YJ, Lee HM, Lee HS, et al. In vivo cortical spreading pattern of tau and amyloid in the Alzheimer disease spectrum. Ann Neurol. 2016 Aug;80(2):247-58.

34. Baker SL, Maass A, Jagust WJ. Considerations and code for partial volume correcting $[18 \mathrm{~F}]-\mathrm{AV}-1451$ tau PET data. Data in brief. 2017;15:648-57.

35. Smith R, Schöll M, Leuzy A, Jögi J, Ohlsson T, Strandberg O, et al. Head-to-head comparison of tau positron emission tomography tracers [18F] flortaucipir and [18F] RO948. Eur J Nucl Med Mol Imaging. 2019:1-3.

36. Otto M. Tau CSF proteins for diagnosis but tau PET imaging for AD diagnosis and staging. Neurology. 2018;10:212.
37. Yoshiyama Y, Lee VM, Trojanowski JQ. Therapeutic strategies for tau mediated neurodegeneration. J Neurol Neurosurg Psychiatry. 2013;84:784-95.

38. Hansson O, Grothe MJ, Strandberg TO, Ohlsson T, Hägerström D, Jögi J et al. Tau pathology distribution in Alzheimer's disease corresponds differentially to cognition-relevant functional brain networks. Front Neurosci. 2017;11:167-.

39. Franzmeier N, Rubinski A, Neitzel J, Kim Y, Damm A, Na DL, et al. Functional connectivity associated with tau levels in ageing, Alzheimer's, and small vessel disease. Brain. 2019;142(4):1093107.

40. Maass A, Landau S, Baker SL, Horng A, Lockhart SN, La Joie R, et al. Comparison of multiple tau-PET measures as biomarkers in aging and Alzheimer's disease. Neuroimage. 2017;157:448-63.

41. Johnson KA, Schultz A, Betensky RA, Horng A, Lockhart SN, La Joie R, et al. Tau positron emission tomographic imaging in aging and early Alzheimer disease. Ann Neurol. 2016;79:110-9.

42. Jagust W. Imaging the evolution and pathophysiology of Alzheimer disease. Nat Rev Neurosci 2018;19:687-.

43. Buckley RF, Mormino EC, Rabin JS, Hohman TJ, Landau S, Hanseeuw BJ, et al. Sex differences in the association of global amyloid and regional tau deposition measured by positron emission tomography in clinically normal older adults. JAMA Neurol. 2019;76:542-51.

44. Laws KR, Irvine K, Gale TM. Sex differences in Alzheimer's disease. Curr Opin Psy. 2018;31:133-9.

45. Fisher DW, Bennett DA, Dong H. Sexual dimorphism in predisposition to Alzheimer's disease. Neurobiol Aging. 2018;70:308-24.

46. Brinton RD, Yao J, Yin F, MackWJ, Cadenas E. Perimenopause as a neurological transition state. Nat Rev Endocrinol 2015;11:393405.

47. Serrano-Pozo A, Qian J, Monsell SE, Betensky RA, Hyman BT. APOE $\varepsilon 2$ is associated with milder clinical and pathological Alzheimer disease. Ann Neurol. 2015;77:917-29.

48. Tzioras M, Davies C, Newman A, Jackson R, Spires-Jones T. Invited review: APOE at the interface of inflammation, neurodegeneration and pathological protein spread in Alzheimer's disease. Neuropath App Neurobiol. 2019;45:327-46.

49. Tromp D, Dufour A, Lithfous S, Pebayle T, Després O. Episodic memory in normal aging and Alzheimer disease: insights from imaging and behavioral studies. Ageing Res Rev. 2015;24:232-62.

50. Cabeza R, Dennis NA. Frontal lobes and aging. Principles of frontal lobe function. $2 d$ ed. New York: Oxford University Press. p. 2012: $628-652$.

51. Jagust W. Vulnerable neural systems and the borderland of brain aging and neurodegeneration. Neuron. 2013;77:219-34.

52. Braak H, Braak E. Neuropathological stageing of Alzheimerrelated changes. Acta Neuropathol. 1991;82:239-59.

53. Farrer LA, Cupples LA, Haines JL, Hyman B, Kukull WA, Mayeux $\mathrm{R}$, et al. Effects of age, sex, and ethnicity on the association between apolipoprotein E genotype and Alzheimer disease: a meta-analysis. Jama. 1997;278:1349-56.

54. Comon P. Independent component analysis, a new concept? Signal Proc. 1994;36:287-314.

55. Bischof GN, Ewers M, Franzmeier N, Grothe MJ, Hoenig M, Kocagoncu E, et al. Connectomics and molecular imaging in neurodegeneration. Eur J Nucl Med Mol Imaging. 2019;11:1-2.

Publisher's note Springer Nature remains neutral with regard to jurisdictional claims in published maps and institutional affiliations. 